\title{
Usefulness of dermoscopy in the diagnosis and monitoring treatment of demodicidosis
}

\author{
Paula Friedman ${ }^{1}$, Emilia Cohen Sabban ${ }^{1}$, Horacio Cabo ${ }^{1}$
}

1 Dermatology Department, Instituto de Investigaciones Médicas “A. Lanari”, University of Buenos Aires, Argentina

\begin{abstract}
Key words: dermoscopy, demodicidosis, standardized skin surface biopsy
Citation: Friedman P, Cohen Sabban E, Cabo H. Usefulness of dermoscopy in the diagnosis and monitoring treatment of demodicidosis. Dermatol Pract Concept. 2017;7(1):6. DOI: https://doi.org/10.5826/dpc.0701a06
\end{abstract}

Received: August 13, 2016; Accepted: October 17, 2016; Published: January 31, 2017

Copyright: @2017 Friedman et al. This is an open-access article distributed under the terms of the Creative Commons Attribution License, which permits unrestricted use, distribution, and reproduction in any medium, provided the original author and source are credited.

Funding: None.

Competing interests: The authors have no conflicts of interest to disclose.

All authors have contributed significantly to this publication.

Corresponding author: Paula Friedman, MD, 2562 Arcos St, 2nd floor, Buenos Aires, Argentina. Tel. +0541158406476. Email: paufriedman@gmail.com

ABSTRACT Demodicidosis is a common infestation and should be considered in the differential diagnosis of recurrent or recalcitrant perioral dermatitis or rosacea-like eruptions of the face. We report on a 34-year-old male, who presented with facial erythema and desquamation accompanied by a pruritic sensation. Dermoscopic examination revealed Demodex tails and Demodex follicular openings, both specific features of this entity. Microscopically, standardized skin surface biopsy test was pathogenic and the patient had positive response to anti-demodectic drugs.

To our knowledge, a few reports of the dermatoscopic features of demodicidosis have been published in the literature. Dermoscopy offers a potential new option for a real-time validation of Demodex infestation and a useful tool for monitoring treatment.

\section{Case Report}

A 34-year-old male patient with no relevant medical history presented at the hospital with facial erythema and desquamation accompanied by a pruritic sensation that had appeared two years before. He had been treated with topical corticosteroids and pure aloe vera for a long time. Physical examination revealed facial keratotic scaly changes with a faint erythematous background on both cheeks and the frontotemporal area (Figures 1,2). At polarized-light dermoscopy, Demodex tails and Demodex follicular openings, erythema and non-specific scales were observed (Figures 3, 4, 5). We performed a cyanoacrylate standardized skin surface biopsy (SSSB) in the cheek involved, and the microscopic examination confirmed the presence of multiple viable Demodex mites (Figure 6). Topical steroids were tapered, and aloe vera was discontinued. The patient was given ivermectin $1 \%$ cream at night, and a significant improvement was seen after two weeks (Figures 7,8). Based on the clinical presentation, positive SSSB examination, and positive response to anti-Demodex therapy, we concluded this was a case of topical steroid induced-demodicosis.

\section{Comments}

Human demodicosis (DD) is a skin disease of the pilosebaceous units associated with human Demodex, a widely 


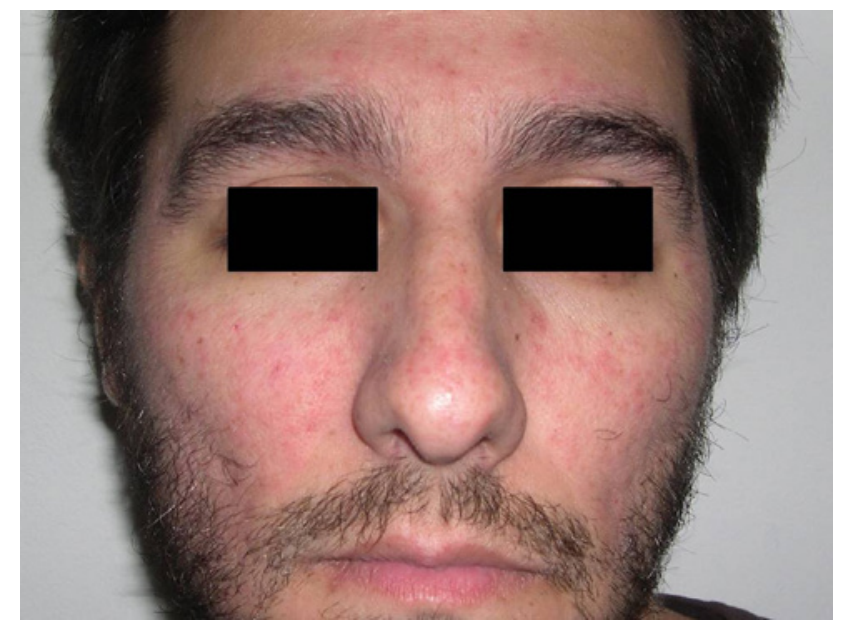

Figure 1. Facial spinulosis (roughness) over an erythematous background. [Copyright: @2017 Friedman et al.]

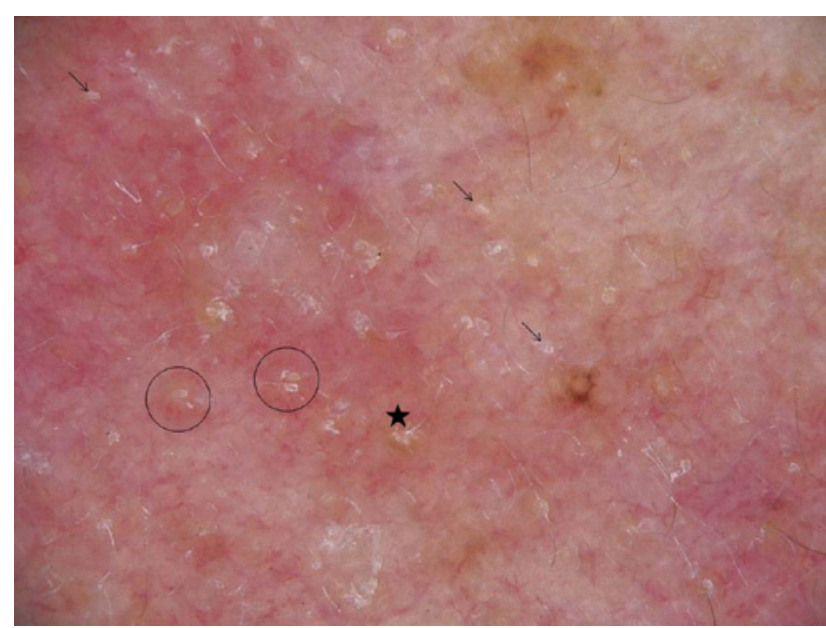

Figure 3. Dermoscopic picture: Demodex "tails" (arrow), Demodex "follicular openings" (star), filaments protruding out of follicular openings (circle), erythema and non-specific scales. [Copyright: (C)2017 Friedman et al.]

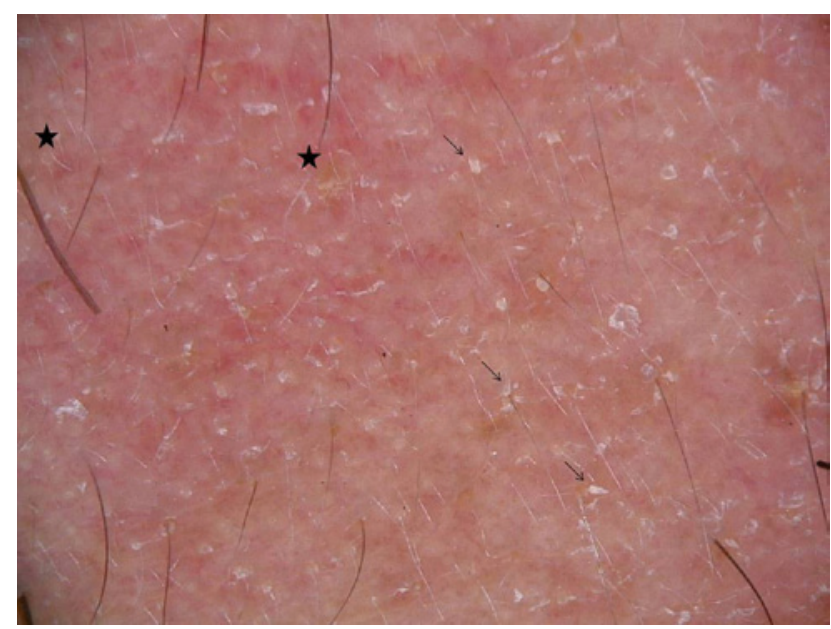

Figure 5. Dermoscopic picture: Demodex "tails" (arrow), Demodex "follicular openings" (star). [Copyright: (C2017 Friedman et al.]

known ectoparasitic mite, involving mainly the face and head. Although it is controversial, this mite is thought to play a pathogenic role in humans. Symptoms may develop when the

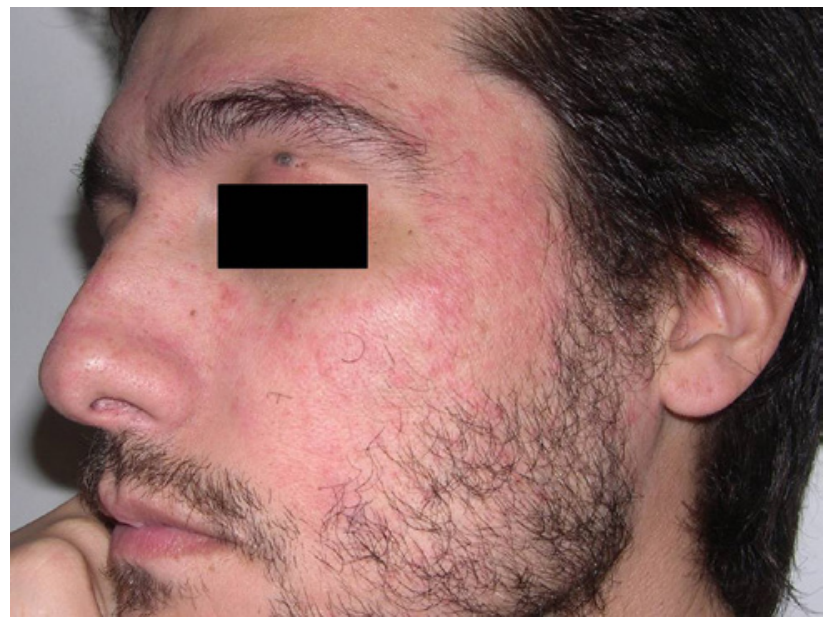

Figure 2. Facial erythema and desquamation on both cheeks and frontotemporal area. [Copyright: @2017 Friedman et al.]

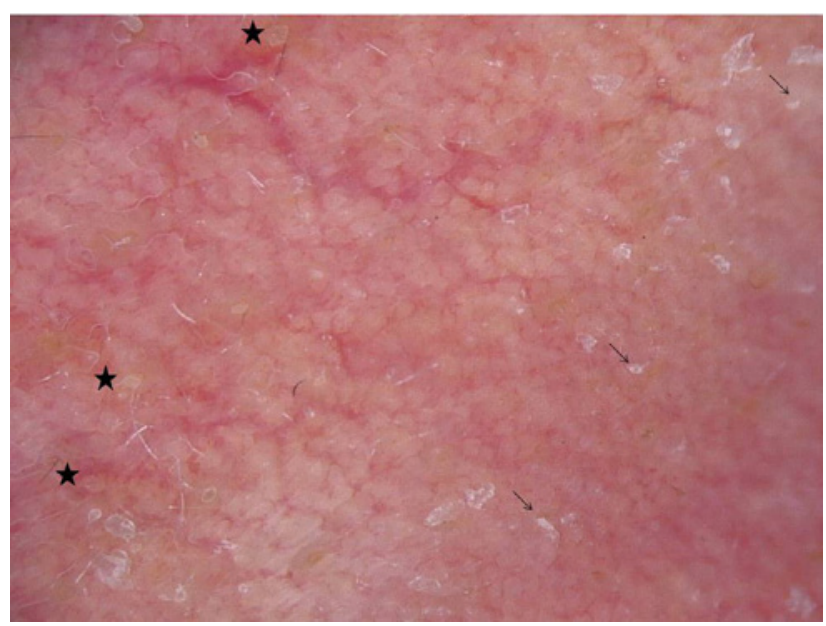

Figure 4. Dermoscopic picture: Demodex "tails" (arrow), Demodex "follicular openings" (star) and non-specific scales. [Copyright: (C)2017 Friedman et al.]

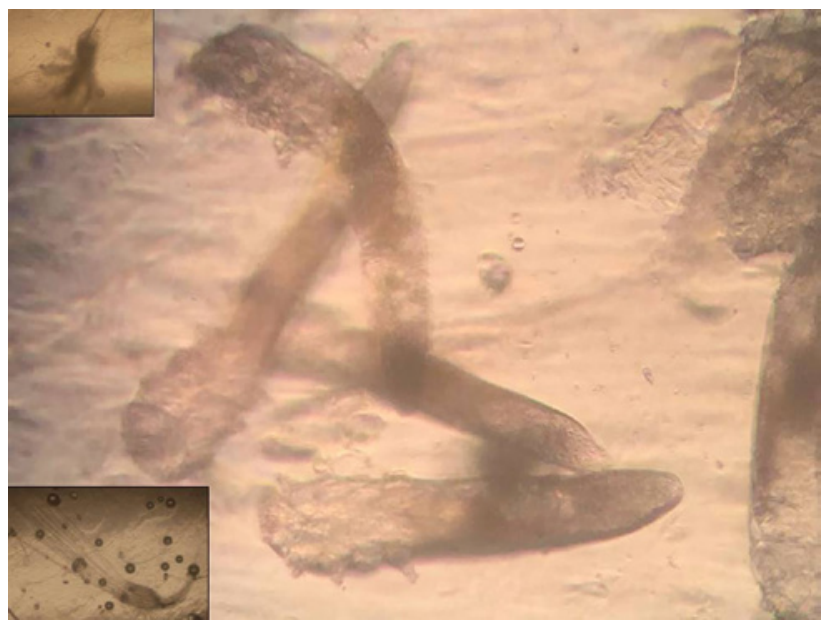

Figure 6. Standardized skin surface biopsy. Microscopic visualization of Demodex folliculorum (original magnification 40x). [Copyright: (02017 Friedman et al.]

follicles become heavily infested, or when the mites penetrate the dermal tissue [1,2].

Clinical manifestations include a group of eruptions 


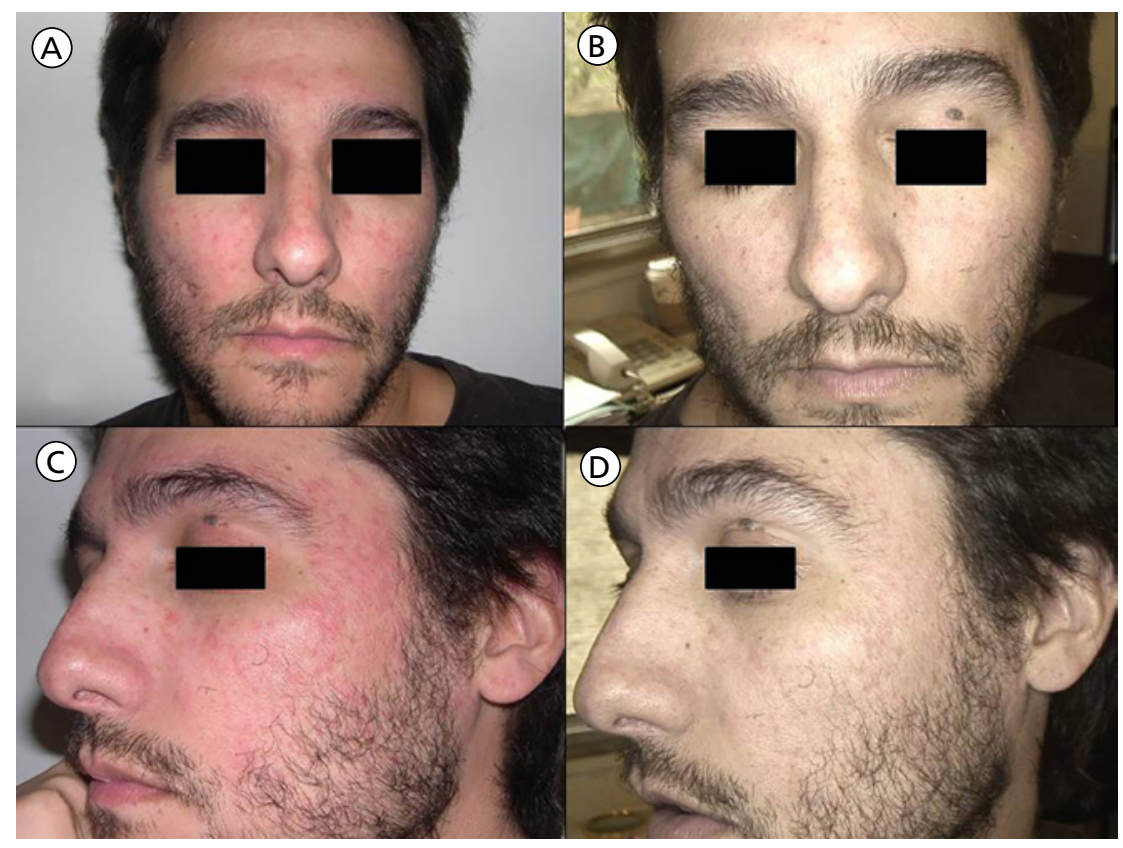

Figure 7. (a) (c) Secondary demodicosis induced by topical corticosteroid treatment. (b) (d) Rash resolved after two weeks of ivermectin 1\% cream at night and a gradual tapering of topical steroid therapy. [Copyright: @2017 Friedman et al.]

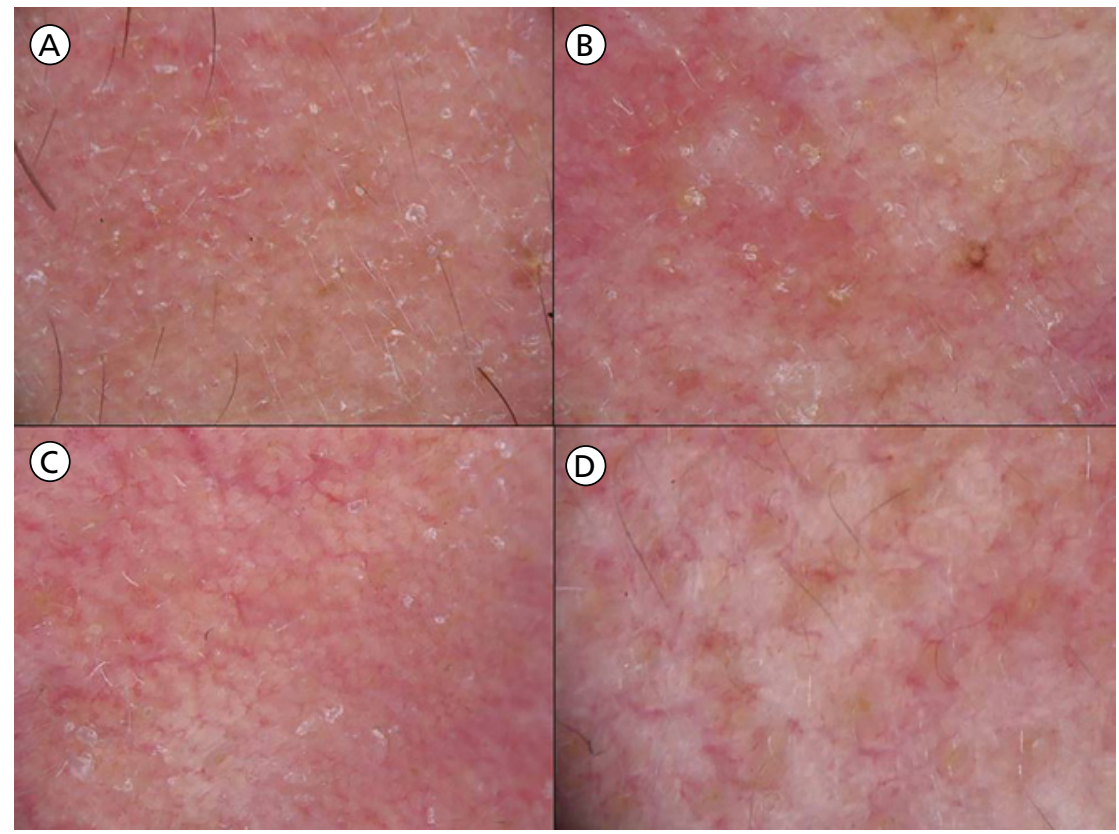

Figure 8. Dermoscopic pictures before (a) (b) (c) and after treatment (d). [Copyright: @2017 Friedman et al.]

characterized by variable degrees of spinulosis (roughness of the skin), erythema, papules, and pustules, usually accompanied by a burning or pruritic sensation. Primary DD is characterized by the absence of preexisting or concurrent inflammatory dermatoses. An abnormal increase of Demodex mites in patients with other known dermatoses or diseases can be classified as secondary DD (Table 1). Our patient had been copy could have helped in demodicidosis recognition, since the patient was incorrectly treated with topical steroids possibly with the diagnosis of seborrheic dermatitis. However, when we evaluated the patient, dermoscopy did not reveal what would be expected for seborrheic dermatitis (dotted vessels in a patchy distribution and fine yellowish scales), but revealed, instead, features associated with demodicidosis ("Demodex tails" and "Demodex follicular openings").

The diagnosis of DD should be made based on three major criteria: the specific clinical presentation, the microscopic observation of a high density of mites and a positive response to antidemodectic drugs [6].

Under dermoscopy, we observed non-follicular and perifollicular gelatinous threads or filaments protruding out of follicular openings known as "Demodex tails." They account for the presence of the mite itself. Demodex follicular openings were also identified as dilated follicular openings containing round, amorphic, grayish/light brown plugs surrounded by an erythematous halo. They are both specific features of DD.

The density of Demodex mites can be studied by SSSB, potassium hydroxide examination, skin biopsy, or a combination of these. SSSB is considered to be the gold standard for diagnosis; with this method, the superficial parts of the horny follicle layer are collected and live mites can be observed on microscopic examination. A density of more than 5 mites/ follicles or 5 mites/cm 2 of SSSB specimen is considered to be pathogenic [7].

Various therapeutic regimens have been proposed to treat DD, including acaricides-ivermectin, permethrin, crotamiton, lindano-and adjuvants, such as systemic and topical metronidazole, salicylic acid, gamma benzene hexachloride, sublimed sulfur and benzyl benzoate. Both effectiveness and optimal dosage still remain to be determined. Our patient was treated with ivermectin $1 \%$ cream at night for two weeks, and both physical and dermoscopic examination improved. 
TABLE 1. Secondary Demodicosis Associations.

[Copyright: (O2017 Friedman et al.]

\begin{tabular}{|l|l|}
\hline Acne & Phototherapy \\
Perioral dermatitis & Melanocytic nevi \\
Papulopustular rosacea & Eyelid basal cell carcinoma \\
Seborrheic dermatitis & Mycosis fungoides \\
Calcineurin inhibitors & Systemic diseases (chronic renal failure) \\
Topical corticosteroids & Leukemia \\
Epidermal growth factor receptor inhibitors & HIV infection \\
\hline
\end{tabular}

TABLE 2. Differential Diagnosis for Demodicosis-Dermoscopic Clues. [Copyright: @2017 Friedman et al.]

\begin{tabular}{|l|l|l|}
\hline \multicolumn{1}{|c|}{ Demodicidosis } & \multicolumn{1}{|c|}{ Seborrheic Dermatitis } & \multicolumn{1}{c|}{ Rosacea } \\
"Demodex tails" & $\begin{array}{l}\text { Dotted vessels in a patchy } \\
\text { distribution } \\
\text { Fine yellowish scales }\end{array}$ & $\begin{array}{l}\text { Erythematotelangiectatic rosacea } \\
\text { Linear vessels characteristically arranged in } \\
\text { a polygonal network } \\
\text { Papulopustular rosacea } \\
\text { Linear vessels characteristically arranged in } \\
\text { a polygonal network } \\
\text { Follicular pustules }\end{array}$ \\
\hline
\end{tabular}

\section{Conclusion}

Dermoscopy may serve as a useful and non-invasive tool for the real-time identification of Demodex infestation, evaluation and follow-up.

\section{References}

1. Donnelly A, Kenney A, DiCaudo D. Demodicosis: clinical, dermatoscopic, and microscopic correlation. [Abstract]. J Am Acad Dermatol. 2013;68(4):Suppl 1,AB117.

2. Hsu CK, Hsu MM, Lee JY. Demodicosis: a clinicopathological study. J Am Acad Dermatol. 2009;60:453-462.
3. Chen W, Plewig G. Human demodicosis: revisit and a proposed classification. Br J Dermatol. 2014;170(6):1219-1225.

4. Kaur T, Jindal N, Bansal R. Facial demodicidosis: a diagnostic challenge. Indian J Dermatol. 2012;57(1):72-73.

5. Errichetti E, Stinco G. Dermoscopy in general dermatology: a practical overview. Dermatol Ther (Heidelb). 2016;6:471.

6. Segal R, Mimouni D, Feuerman H. Dermoscopy as a diagnostic tool in demodicidosis. Int J Dermatol. 2010;49(9):1018-1023.

7. Aşkın Ü, Seckin D. Comparison of the two techniques for measurement of the density of Demodex folliculorum: standardized skin surface biopsy and direct microscopic examination. Br J Dermatol. 2010;162(5):1124-1126. 